\title{
Nano Copper Ferrite Catalyzed Sonochemical, One-Pot Three and Four Component Synthesis of Poly Substituted Imidazoles
}

\author{
Paul D. Sanasi*, Ravi K. Majji, Swathi Bandaru, Satyanarayana Bassa, Surekha Pinninti, \\ Sruthi Vasamsetty, Raghu B. Korupolu \\ Department of Engineering Chemistry, A. U. College of Engineering (A), Andhra University, Visakhapatnam, \\ India \\ Email: *pauldouglas12@gmail.com
}

Received 9 November 2015; accepted 10 January 2016; published 13 January 2016

Copyright (C) 2016 by authors and Scientific Research Publishing Inc.

This work is licensed under the Creative Commons Attribution International License (CC BY).

http://creativecommons.org/licenses/by/4.0/

(c) (i) Open Access

\begin{abstract}
A simple, multi component, one-pot method has been reported for the synthesis of poly substituted imidazoles in presence of magnetically separable and recyclable spinel nano copper ferrite as heterogeneous catalyst by the cyclo-condensation of benzil, aromatic aldehyde, ammonium acetate and substituted amines under ultrasonic irradiation. This method of preparation has many advantages compared to those methods which are previously reported in the literature. This methodology offers simple experimental procedure, milder reaction conditions and environmentally benign approach.
\end{abstract}

\section{Keywords}

Multi Component Reaction, One-Pot Synthesis, Nano Copper Ferrite Catalyst, Poly Substituted Imidazoles, Ultrasonic Irradiation

\section{Introduction}

One-pot, multi component reactions (MCRs) have significant importance due to formation of a single product with high yields by the combination of two or more components in a single step process [1] [2]. This method is a convenient and great technique for the preparation of some biologically and medicinally active pharmaceutical

"Corresponding author.

How to cite this paper: Sanasi, P.D., Majji, R.K., Bandaru, S., Bassa, S., Pinninti, S., Vasamsetty, S. and Korupolu, R.B. (2016) Nano Copper Ferrite Catalyzed Sonochemical, One-Pot Three and Four Component Synthesis of Poly Substituted Imidazoles. Modern Research in Catalysis, 5, 31-44. http://dx.doi.org/10.4236/mrc.2016.51004 
ingredients.

Imidazole derivatives are very interesting class of Nitrogen containing 5-membered heterocyclic compounds. Because of their wide range of pharmacological importance and significant role in biochemical processes [3] [4], the Biological importance of the poly substituted imidazole ring system has made it a common structure in numerous synthetic compounds, such as fungicides [5], herbicides [5], therapeutic agents [6] and plant-growth regulators [7]. This core also has been utilized in diverse pharmaceutical applications such as anti-inflammatory [8], anti-thrombotic [9] and antitumor [10] agents. Due to their great biological importance of poly substituted imidazole framework, the synthesis of Poly substituted imidazoles has attracted much attention in organic synthesis.

Owing to their enormous collection of pharmacological and biological activities many synthetic strategies has been developed for synthesis of substituted imidazoles such as the hetero-cope rearrangement [11], four component condensation of arylglyoxals, primary amines, carboxylic acids and isocyanides on wangresin [12] and three component condensation of $\alpha$-azido chalcones, aromatic aldehyde and substituted aniline in presence of Erbiumtriflate [13]. Later on a first, simple and convenient synthetic methodology has been reported by Radziszewski and japp for the synthesis of 2,4,5-tri phenyl imidazoles by using 1,2 dicarbonyl compounds, ammonia, various aldehydes [14]. Afterward several methods have been reported for the synthesis of tri-substituted imidazoles by cyclo condensation of 1,2 dicarbonyl compounds, various aromatic aldehydes and ammonium acetate using various catalysts such as $\mathrm{NiCl}_{2} \cdot 6 \mathrm{H}_{2} \mathrm{O} / \mathrm{Al}_{2} \mathrm{O}_{3}$ [15], $\mathrm{MoO}_{3} / \mathrm{SiO}_{2}$ [16], Polymer supported zinc chloride [17], trichloro isocyanuric acid (TCCA) [18] under reflux conditions and glyoxalic acid as catalyst under microwave conditions [19] and Zinc (II) (tetra (4-methylphenyl)) porphyrin [20], Magnetic $\mathrm{Fe}_{3} \mathrm{O}_{4}$ nano particles [21], Sulfamic acid functionalized magnetic nano particles SA-MNP [22], Ionic liquid (EMIM)OAc [23], $\operatorname{Zr}(\mathrm{acac})_{4}$ [24], ceric ammonium nitrate [25] as catalysts under ultra sound assisted conditions. In the same way several methods have been reported for the synthesis of tetra substituted imidazoles by cyclo condensation of 1,2 dicarbonyl compounds, various aromatic aldehydes, ammonium acetate and aromatic/aliphatic amines using various catalysts such as nano $\mathrm{TiCl}_{4} \cdot \mathrm{SiO}_{2}$ [26], $\mathrm{FeCl}_{3} \cdot 6 \mathrm{H}_{2} \mathrm{O}$ [27], $\mathrm{BF}_{3} \cdot \mathrm{SiO}_{2}$ [28], $\mathrm{HClO}_{4}-\mathrm{SiO}_{2}$ [29], Keggin type heteropoly acid $\mathrm{H}_{4}\left[\mathrm{PMo}_{11} \mathrm{VO}_{40}\right]$ [30] under reflux conditions and Potassium dodecatugstocobaltate trihydrate $\left(\mathrm{K}_{5} \mathrm{CoW}_{12} \mathrm{O}_{40} \cdot 3 \mathrm{H}_{2} \mathrm{O}\right)$ [31], zeolite $\mathrm{HY}$ and silica gel [32] as catalyst under microwave conditions and nano crystalline $\mathrm{MgAl}_{2} \mathrm{O}_{4}$ [33] as catalyst under Ultra sound irradiation. In extension with these procedures, several methods have been reported for the synthesis of both tri and tetra substituted imidazoels using various catalysts such as molecular Iodine [34], Sodium dihydrogen phosphate [35], $\mathrm{BiCl}_{3}$ [36], DABCO [37], Sulfonic acid functionalized SBA-15 nanoporous material [SBA-Pr-SO ${ }_{3} \mathrm{H}$ ] [38], L-proline [39], nano crystalline sulphated zirconia [40], $\mathrm{InCl}_{3} \cdot 3 \mathrm{H}_{2} \mathrm{O}$ [41] under reflux conditions and Well-Dawson heteropolyacid $\left(\mathrm{H}_{6} \mathrm{P}_{2} \mathrm{~W}_{18} \mathrm{O}_{62} \cdot 24 \mathrm{H}_{2} \mathrm{O}\right)$ supported on silica [42] under microwave conditions.

According to the literature survey, several synthetic protocols have been reported for the synthesis of substituted imidazoles using various type of copper containing catalyst such as synthesis of imidazoles through the $\mathrm{Cu}_{2} \mathrm{O}$ catalyzed cross-cycloaddition between two different isocyanides [43], Synthesis of 1,2,4-trisubstituted imidazoles through the $\mathrm{CuCl}_{2}$-catalyzed oxidative diamination of terminal alkynes by amidines [44], Synthesis of multi substituted imidazoles via CuI-catalyzed [3 + 2] cycloadditions [45]. The above reported methods have its own importance and merits, however most of these methods require harmful catalysts, and difficult work-up and effluent pollution. Therefore, development of environmentally benign, green procedure for preparation of poly substituted imidazoles is highly desirable.

Due to the effective activity of magnetically separable copper based nano particles [46], several organic transformations [47] are carried out with these particles and reported earlier in the literature. Along with these, nano copper ferrite has earlier been used as magnetically separable catalyst for several organic synthetic reactions such as asymmetric hydrosilylation of Ketones [48], synthesis of diaryl or aryl akyl sulfides via cross coupling process under ligand free conditions [49], synthesis of substituted benzoxazoles via Ullmann-type coupling under ligand free conditions [50], Cross-coupling of aryl halides with diphenyl diselenide [51], green one-pot three component synthesis of spirooxindoles [52], multicomponent synthesis of 1,4-di substituted 1,2,3triazoles in tap water [53].

Ultrasound-Assisted organic synthesis (UAOS) has been increasingly developed by researchers across the globe for the synthesis of organic molecules since last three decades. Ultrasound irradiation offers an alternative energy source for organic reactions which are ordinarily accomplished by heating. The salient features of Ultrasonic irradiation are enhanced reaction rates, formation of purer products, easier manipulation, energy conservation, waste minimization and this technique is more convenient to taking green chemistry concepts into an ac- 
count [54] [55].

Ultrasound-assisted reactions proceed by the formation, growth and collapse of acoustic bubbles in the reaction medium. These directly help in shortening the time span of reactions and increasing the yield of products [56]. Moreover a large number of reactions can be carried out in shorter reaction time, higher yield through milder reaction conditions under Ultra sound Irradiation [57]-[59]. Many homogeneous and heterogeneous reactions can be conducted smoothly by sonication to provide improved yields and increased selectivity [60].

As a part of our ongoing research towards the synthesis of biologically active heterocyclic compounds using magnetically separable nano catalysts, keeping environmental friendly methods in mind, here we report an efficient and simple work-up method for the synthesis of poly substituted imidazoles using magnetic separable nano copper ferrite as heterogeneous catalyst under ultrasonic irradiation.

\section{Experimental}

\subsection{Chemicals and Apparatus}

All chemicals are purchased from commercial sources and liquid aromatic aldehydes and liquid aromatic amines are purified by distillation prior to use. Melting points of the products were recorded on an electrochemical apparatus and they were compared with literature values. ${ }^{1} \mathrm{HNMR}$ and ${ }^{13} \mathrm{CNMR}$ spectral data were performed on the Bruker-Avance $400 \mathrm{MHz}$ and $100 \mathrm{MHz}$ spectrometers respectively in DMSO- $\mathrm{d}_{6} / \mathrm{CDCl}_{3}$. The chemical shift values were reported on the $\delta$ scale in parts per million (ppm), downfield from tetramethylsilane (TMS) as an internal standard. IR Spectra were recorded in KBr disks with a Bruker $\alpha$-FTIR Spectrometer. The mass spectrum was recorded using a Perkin-Elmer PE SCIEX-API 2000, equipped with ESI source used online with a HPLC system after the ultraviolet (UV) detector.

Ultrasonication was performed in a Rivotek ultrasonic cleaner with Transducer PZT crystals (Morgan Matrac, UK make) bonded on the base of the tank with welbond technique (frequency of $40 \mathrm{kHz}$ and an output power of $250 \mathrm{~W}$ ). The Flask containing the reaction mixture was located in the maximum energy area in the water bath where the surface of the reactants (reaction vessel) is slightly lower than the level of the water and the addition and removal of water controlled the temperature of the water bath.

\subsection{Catalyst Preparation}

The spinel nano copper ferrite has been synthesized by citrate sol-gel precursor method as reported earlier by us [46].

\subsection{Catalyst Characterization}

The catalyst has been characterized using FTIR, SEM, TEM, XRD and particle size analyzer as reported earlier [46].

\subsection{General Procedure for the Synthesis of 2,4,5-Tri Substituted Imidazoles}

Benzil (10 mmol), aromatic aldehyde $(10 \mathrm{mmol})$, ammonium acetate $(20 \mathrm{mmol})$ and Copper ferrite $(2 \mathrm{mmol})$ were taken in a flask and the contents are dissolved in $5 \mathrm{~mL}$ of ethanol. Then the reaction mixture was sonicated at room temperature for prescribed time (Scheme 1). The progress of the reaction was monitored by TLC (n-hexane: ethyl acetate 4:1). After completion of the reaction the catalyst was separated from the reaction mixture by using an external magnet and then the reaction mixture was concentrated in a rotary evaporator to remove the solvent. Then the dried product was recrystallised from hot ethanol for several times to get the corresponding pure product. The products were confirmed by IR, NMR, Mass spectra and by melting points.

\subsection{General Procedure for the Synthesis of 1,2,4,5-Tetra Substituted Imidazoles}

Benzil $(10 \mathrm{mmol})$, aromatic aldehyde $(10 \mathrm{mmol})$, ammonium acetate $(10 \mathrm{mmol})$, aromatic amine $(10 \mathrm{mmol})$ and Copper ferrite $(2 \mathrm{mmol})$ were taken in a flask and the contents are dissolved in $5 \mathrm{~mL}$ of ethanol. Then the reaction mixture was sonicated at $40^{\circ} \mathrm{C}$ for prescribed time (Scheme 2). The progress of the reaction was monitored by TLC (n-hexane: ethyl acetate 4:1). After completion of the reaction the catalyst was separated from the reaction mixture by using an external magnet and then the reaction mixture was concentrated in Rotavapour to removal of the solvent. Then the dried product was recrystallised from hot ethanol for several times to get the corresponding pure product. The products were confirmed by IR, NMR, Mass spectra and by melting points. 


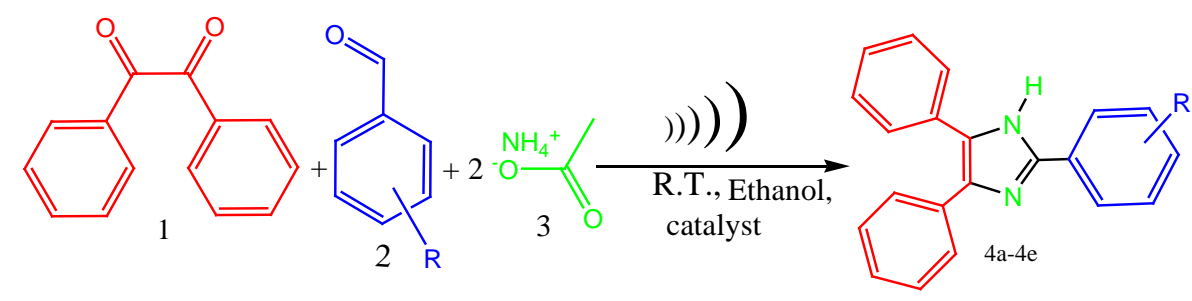

$\mathrm{R}=$ a) m-Br, b) p-F, c) m-NO $\mathrm{NO}_{2}$, $)$ p-NO $\mathrm{NO}_{2}$, e) p- $\mathrm{CH}_{3}$

Scheme 1. A generalized scheme for synthesis of 2,4,5-tri substituted imidazoles in presence of copper nano ferrite by cyclocondensation of benzil, aromatic aldehyde and ammonium acetate under ultra sound irradiation.<smiles>[R]c1cccc(-c2nc(-c3ccccc3)c(-c3ccccc3)n2[R1])c1</smiles>

a) $\mathrm{R}=\mathrm{H} \mathrm{R}^{1}=\mathrm{C}_{6} \mathrm{H}_{5}$ b) $\left.\mathrm{R}=\mathrm{p}-\mathrm{OCH}_{3}, \mathrm{R}^{1}=\mathrm{C}_{6} \mathrm{H}_{5}, \mathrm{c}\right) \mathrm{R}=\mathrm{H}, \mathrm{R}^{1}=\mathrm{C}_{6} \mathrm{H}_{5}-\mathrm{CH}_{2}$ d) $\mathrm{R}=\mathrm{p}-\mathrm{OCH}_{3}, \mathrm{R}^{1}=\mathrm{C}_{6} \mathrm{H}_{5}-\mathrm{CH}_{2}$ e) $\mathrm{R}=\mathrm{p}-\mathrm{CH}_{3}, \mathrm{R}^{1}=\mathrm{C}_{6} \mathrm{H}_{5}-\mathrm{CH}_{2}$ f) $\mathrm{R}=\mathrm{p}-\mathrm{Cl}, \mathrm{R}^{1}=\mathrm{C}_{6} \mathrm{H}_{5}-\mathrm{CH}_{2}$ g) $\mathrm{R}=\mathrm{p}-\mathrm{Cl}, \mathrm{R}^{1}=$ cyclohexyl h) $\mathrm{R}=\mathrm{p}-\mathrm{CH}_{3}, \mathrm{R}^{1}=$ cyclohexyl

Scheme 2. A generalized scheme for synthesis of 1,2,4,5-tetra substituted imidazoles in presence of catalyst by cyclo condensation of benzil, aromatic aldehyde, ammonium acetate and substituted amine under ultra sonic irradiation.

\subsection{Spectra Data}

\subsubsection{Spectral Data for $2,4,5$-Tri Substituted Imidazoles}

\begin{tabular}{cc}
\hline Compound & Spectral data \\
\hline
\end{tabular}

2-(3-Bromo phenyl)-4,5,diphenyl-1-H Imidazole: (4a)<smiles>Brc1cccc(-c2nc(-c3ccccc3)c(-c3ccccc3)[nH]2)c1</smiles>

2-(4-Flouro phenyl)-4,5,diphenyl-1-H Imidazole: (4b)<smiles>Fc1ccc(-c2nc(-c3ccccc3)c(-c3ccccc3)[nH]2)cc1</smiles>

2-(3-Nitro phenyl)-4,5,diphenyl-1-H Imidazole: (4c)<smiles>O=[N+]([O-])c1cccc(-c2nc(-c3ccccc3)c(-c3ccccc3)[nH]2)c1</smiles>

M.P. $300^{\circ} \mathrm{C}-302^{\circ} \mathrm{C}$; ${ }^{1} \mathrm{HNMR}$ (Bruker) $\left(400 \mathrm{MHz}, \mathrm{CDCl}_{3} / \mathrm{DMSO}_{6} \mathrm{~d}_{6}\right.$ ):

$\delta=7.22-7.48(\mathrm{~m}, 10 \mathrm{H}), 7.65(\mathrm{~s}, 1 \mathrm{H}), 7.42(\mathrm{~d}, 1 \mathrm{H}), 7.39(\mathrm{~d}, 1 \mathrm{H}), 7.21(\mathrm{t}, 1 \mathrm{H})$; ${ }^{13} \mathrm{CNMR}\left(100 \mathrm{MHz}, \mathrm{CDCl}_{3} / \mathrm{DMSO}_{\mathrm{d}}\right.$ );

$\delta=123.6,126.5,127.5,128.8,129.3,132.9,147.6$ ppm; FTIR $\left(\mathrm{KBr}, \mathrm{cm}^{-1}\right)$; $3432(\mathrm{~N}-\mathrm{H}), 1600(\mathrm{C}=\mathrm{C}), 1482(\mathrm{C}=\mathrm{N}), 729(\mathrm{C}-\mathrm{Br})$ : NCMS $(\mathrm{m} / \mathrm{z})\left(\mathrm{M}^{+}+1\right) 367$

M.P. $188^{\circ} \mathrm{C}-190^{\circ} \mathrm{C} ;{ }^{1} \mathrm{HNMR}$ (Bruker) $\left(400 \mathrm{MHz} \mathrm{CDCl}_{3} / \mathrm{DMSO}^{-} \mathrm{d}_{6}\right.$ ): $\delta=7.22-7.48(\mathrm{~m}, 10 \mathrm{H}), 7.46-7.42(\mathrm{~d}, \mathrm{~J}=8.0 \mathrm{~Hz}, 2 \mathrm{H})$, 7.03 - 6.71 (d, J = 8.6 Hz, 2H); ${ }^{13} \mathrm{CNMR}\left(100 \mathrm{MHz}, \mathrm{CDCl}_{3} / \mathrm{DMSO}_{\mathrm{d}}\right.$ ); $\delta=126.3,127.5,128.9,129.1,129.3,162.9,133.1$ ppm; FTIR(KBr, $\left.\mathrm{cm}^{-1}\right)$; $3432(\mathrm{~N}-\mathrm{H}), 1600(\mathrm{C}=\mathrm{C}), 1482(\mathrm{C}=\mathrm{N}), 692(\mathrm{C}-\mathrm{F})$ : NCMS $(\mathrm{m} / \mathrm{z}) 315\left(\mathrm{M}^{+}+1\right)$

M.P: $300^{\circ} \mathrm{C}$. FTIR $\left(\mathrm{KBr}, \mathrm{cm}^{-1}\right)$ : 3448, 3068, 1526, 1350;

${ }^{1} \mathrm{HNMR}$ (Bruker) (400 MHz, $\mathrm{CDCl}_{3} / \mathrm{DMSO}^{-} \mathrm{d}_{6}$ ):

$\delta=13.10(\mathrm{~s}, 1 \mathrm{H}), 8.95(\mathrm{~s}, 1 \mathrm{H}), 8.53(\mathrm{~d}, \mathrm{~J} 1 / 47.5 \mathrm{~Hz}, 1 \mathrm{H})$,

$8.23(\mathrm{~d}, \mathrm{~J}=7.8 \mathrm{~Hz}, 1 \mathrm{H}), 7.81$ (d, J1/4 7.8 Hz, 1H),7.54 - 7.33 (m, 10H);

${ }^{13} \mathrm{C}$ NMR (100 MHz, $\mathrm{CDCl}_{3} / \mathrm{DMSO}^{-\mathrm{d}_{6}}$ ):

$\delta=148.4,143.4,131.8,131.2,130.4,128.7,128.4,127.1,122.6,119.4 ;$ $\operatorname{NCMS}(m / z): 342.3\left(\mathbf{M}^{+}+1\right)$ 


\section{Continued}

2-(4-Nitro phenyl)-4,5,diphenyl-1-H Imidazole: (4d)

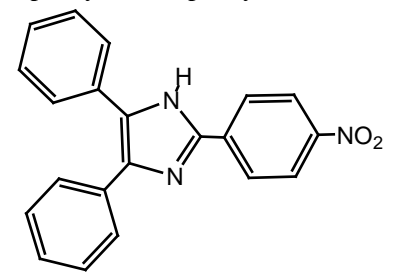

M.P: $199^{\circ} \mathrm{C}-201^{\circ} \mathrm{C}$. FTIR $\left(\mathrm{KBr}, \mathrm{cm}^{-1}\right)$ : 3402, 2928, 1598, 1519, 1346, 856;

${ }^{1} \mathrm{H}$ NMR (Bruker) $\left(400 \mathrm{MHz}, \mathrm{CDCl}_{3} / \mathrm{DMSO}^{-\mathrm{d}_{6}}\right)$ :

$\delta=12.81(\mathrm{~s}, 1 \mathrm{H}), 8.01-7.42(\mathrm{~m}, 14 \mathrm{H})$;

${ }^{13} \mathrm{C}$ NMR (100 MHz, CDCl 3 /DMSO- $\left.\mathrm{d}_{6}\right)$ :

$\delta=148.9,143.7,131.6,130.6,129.7,128.4,127.8,127.4,126.9,126.1$,

125.4, 124.3, 122.2, 118.5; NCMS (m/z): $342.2\left(\mathrm{M}^{+}+1\right)$.

2-(4-Methyl phenyl)-4,5,diphenyl-1-H Imidazole: (4e)

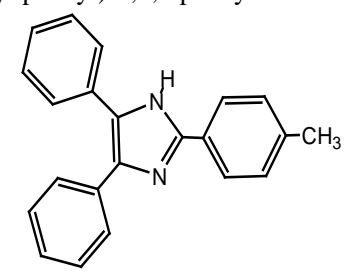

M.P: $184^{\circ} \mathrm{C}-186^{\circ} \mathrm{C} . \operatorname{FTIR}\left(\mathrm{KBr}, \mathrm{cm}^{-1}\right)$ : $3450(\mathrm{~N}-\mathrm{H}), 1600(\mathrm{C}=\mathrm{C}), 1585(\mathrm{C}=\mathrm{N})$. ${ }^{1} \mathrm{HNMR}$ (Bruker) (400 MHz, CDCl $/ \mathrm{DMSO}^{-\mathrm{d}_{6}}$ ):

$2.30\left(\mathrm{~s}, \mathrm{CH}_{3}\right), 7.10-7.60(\mathrm{~m}, 10 \mathrm{H})$,

$7.70(\mathrm{~d}, 2 \mathrm{H}, \mathrm{J}=10 \mathrm{~Hz}), 7.30(\mathrm{~d}, 2 \mathrm{H}, \mathrm{J}=10 \mathrm{~Hz})$

${ }^{13} \mathrm{CNMR}$ (100 MHz, CDCl 3 /DMSO-d D $_{6}$, 55.7, 113.4, 122.6, 126.3,

126.6, 128.0, 128.3, 133.4, 145.7, 159.6 ppm: NCMS $(\mathrm{m} / \mathrm{z}): 311(\mathrm{M}+1)$

\subsubsection{Spectral Data for $2,4,5$-Tri Substituted Imidazoles}

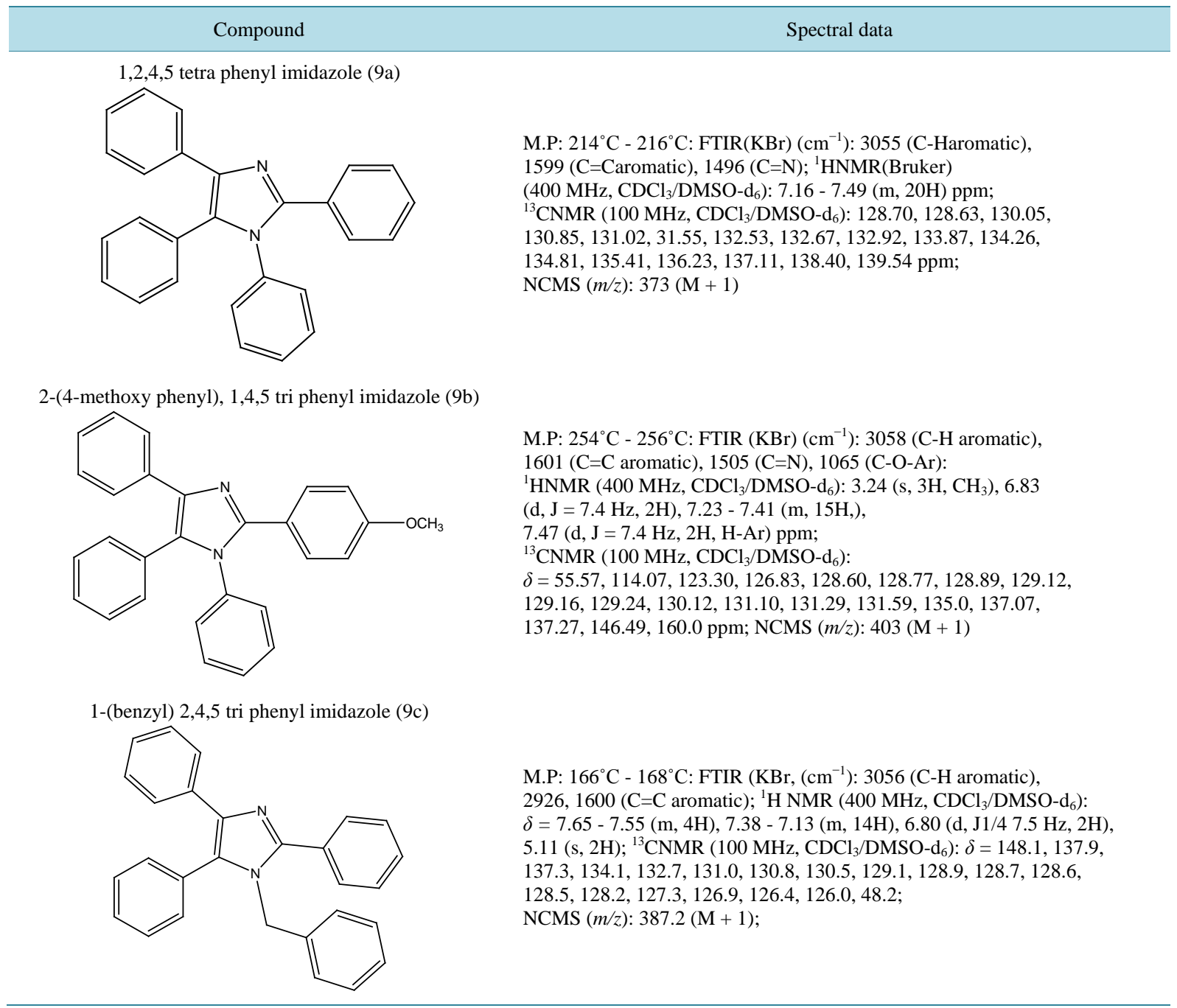




\section{Continued}

1-(benzyl), 2(4-methoxy phenyl), 4,5 di phenyl imidazole (9d)<smiles>COc1ccc(-c2nc(-c3ccccc3)c(-c3ccccc3)n2Cc2ccccc2)cc1</smiles>

1-(benzyl), 2(4-methyl phenyl), 4,5 di phenyl imidazole (9e)<smiles>Cc1ccc(-c2nc(-c3ccccc3)c(-c3ccccc3)n2Cc2ccccc2)cc1</smiles>

1-(benzyl), 2(4-chloro phenyl), 4,5 di phenyl imidazole (9f)<smiles>Clc1ccc(-c2nc(-c3ccccc3)c(-c3ccccc3)n2Cc2ccccc2)cc1</smiles>

1-(cyclohexyl), 2(4-chloro phenyl), 4,5 di phenyl imidazole (9g)<smiles>Clc1ccc(-c2nc(-c3ccccc3)c(-c3ccccc3)n2C2CCCCC2)cc1</smiles>

1-(cyclohexyl), 2(4-methyl phenyl), 4,5 di phenyl imidazole (9h)<smiles>Cc1ccc(-c2nc(-c3ccccc3)c(-c3ccccc3)n2C2CCCCC2)cc1</smiles>

M.P: $158^{\circ} \mathrm{C}-160^{\circ} \mathrm{C}$ : FTIR $\left(\mathrm{KBr},\left(\mathrm{cm}^{-1}\right)\right.$ : 3047 (C-H aromatic), 1604 (C=C aromatic); ${ }^{1} \mathrm{HNMR}\left(400 \mathrm{MHz}, \mathrm{CDCl}_{3} / \mathrm{DMSO}^{\mathrm{d}} \mathrm{d}_{6}\right)$ : d 7.58 (d, J1/4 $7.8 \mathrm{~Hz}, 4 \mathrm{H}), 7.31$ - $7.12(\mathrm{~m}, 11 \mathrm{H}), 6.92-6.80(\mathrm{~m}, 4 \mathrm{H})$,

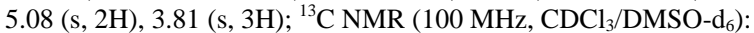
$\delta=159.9,147.9,137.6,134.5,131.0,130.9,130.3,129.7,129.0$, 128.7, 128.5, 127.9, 127.2, 126.7, 126.2, 125.9, 123.3, 113.9, 55.2, 48.1; NCMS (m/z): $417.3(\mathrm{M}+1)$

M.P: $168^{\circ} \mathrm{C}-170^{\circ} \mathrm{C}$ : FTIR (KBr) $\left(\mathrm{cm}^{-1}\right): 3020$ (C-H aromatic), 1600 (C=C aromatic), $1484(\mathrm{C}=\mathrm{N}), 1448$.

${ }^{1} \mathrm{H}$ NMR (400 MHz, CDCl $\left./ \mathrm{DMSO}^{\mathrm{D}} \mathrm{d}_{6}\right): \delta=2.36$ (s, 3H, $\mathrm{CH}_{3}$ ), 5.09 (s, $\left.2 \mathrm{H}, \mathrm{CH}_{2}\right), 6.80-7.59$ (m, 19H, Ar-H) ppm.

${ }^{13} \mathrm{C}$ NMR (100 MHz, CDCl 3 /DMSO- $\mathrm{d}_{6}$ ):

$\delta=159.9,147.9,137.6,134.5,131.0,130.9,130.3,129.7$, 129.0, 128.7, 128.5, 127.9, 127.2, 126.7, 126.2, 125.9, 123.3, 113.9, 55.2, 48.1: NCMS $(\mathrm{m} / \mathrm{z}): 401(\mathrm{M}+1)$

M.P: $162^{\circ} \mathrm{C}-164^{\circ} \mathrm{C}$ : FTIR (KBr) $\left(\mathrm{cm}^{-1}\right): 1600,1477,1447,1414$. ${ }^{1} \mathrm{HNMR}$ (400 MHz, $\left.\mathrm{CDCl}_{3} / \mathrm{DMSO}-\mathrm{d}_{6}\right): \delta=5.08\left(\mathrm{~s}, 2 \mathrm{H}, \mathrm{CH}_{2}\right.$ ), 6.81 - 7.60 (m, 19H, ArH); ${ }^{13} \mathrm{C} \mathrm{NMR} \mathrm{(100} \mathrm{MHz,} \mathrm{CDCl} 3 /$ DMSO-d $\left._{6}\right)$ : $\delta=48.3,125.9,126.5,126.8,127.5,128.1,128.7,128.75,128.8$, $128.85,129.5,130.2,130.4,130.8,131.0,134.3,134.9,137.3$, 138.3, 146.8. NCMS (m/z): $421(\mathrm{M}+1)$

M.P: $152^{\circ} \mathrm{C}-154^{\circ} \mathrm{C}$; FTIR (KBr) $\left(\mathrm{cm}^{-1}\right) 1599,1499,1481,1465$; ${ }^{1} \mathrm{HNMR}\left(400 \mathrm{MHz}, \mathrm{CDCl}_{3} / \mathrm{DMSO}^{\mathrm{d}} \mathrm{d}_{6}\right)$ : $\delta=0.76-1.86\left(\mathrm{~m}, 10 \mathrm{H}, 5\left(\mathrm{CH}_{2}\right), 3.95(\mathrm{t}, 1 \mathrm{H}, \mathrm{CH}), 7.02-7.48\right.$ (m, $14 \mathrm{H}, \mathrm{Ar}-\mathrm{H}) .{ }^{13} \mathrm{CNMR}\left(100 \mathrm{MHz}, \mathrm{CDCl}_{3} / \mathrm{DMSO}^{\mathrm{d}} \mathrm{d}_{6}\right)$ : $\delta=21.32\left(\mathrm{CH}_{3}\right), 24.99,26.12,33.51,58.24,125.85,126.60$, 127.77, 128.52, 128.64, 128.93, 129.45, 129.77, 132.16, 132.62, 134.69, 137.63, 138.62, 147.75. NCMS $(m / z): 413(M+1)$

M.P: $163^{\circ} \mathrm{C}-165^{\circ} \mathrm{C}$; FTIR $(\mathrm{KBr})\left(\mathrm{cm}^{-1}\right)$; 1599, 1499, 1481, 1465; ${ }^{1} \mathrm{HNMR}\left(400 \mathrm{MHz}, \mathrm{CDCl}_{3} / \mathrm{DMSO}^{\mathrm{d}} \mathrm{d}_{6}\right.$ ):

$\delta=0.76-1.86\left(\mathrm{~m}, 10 \mathrm{H}, 5\left(\mathrm{CH}_{2}\right), 2.44\left(\mathrm{~s}, 3 \mathrm{H}, \mathrm{CH}_{3}\right), 3.95(\mathrm{t}, 1 \mathrm{H}, \mathrm{CH})\right.$, 7.02 - 7.48 (m, $14 \mathrm{H}, \mathrm{Ar}-\mathrm{H}) .{ }^{13} \mathrm{CNMR}\left(100 \mathrm{MHz}, \mathrm{CDCl}_{3} /\right.$ DMSO-d $\left._{6}\right)$ : $\delta=21.32\left(\mathrm{CH}_{3}\right), 24.99,26.12$, 33.51, 58.24, 125.85, 126.60, 127.77, 128.52, 128.64, 128.93, 129.45, 129.77, 132.16, 132.62, 134.69, 137.63, 138.62, 147.75. NCMS (m/z): $393(\mathrm{M}+1)$ 


\section{Results and Discussion}

\subsection{Plausible Mechanism for the Formation of Products under Nano Copper Ferrite}

The plausible mechanism for the synthesis of 2,4,5-tri substituted imidazoles [46] with the copper nano ferrite in the reaction may be shown in Scheme 3. Ammonia molecules are obtained from Ammonium acetate. The Aldehyde and 1, 2-diketone are first activated by ferrite nano particles ( $\mathrm{Fe}^{3+}$ ) to afford (A) and (B) respectively. The imine intermediate (A), condenses further with the carbonyl carbon or 1,2 diketone imine (B) and formation of carbocation (C) followed by attack of imine nitrogen to positive centre and dehydration to afford the imo-imidazole (D), which rearranges via 1,5 sigmatropic shift followed by deprotonation gives tri substituted imidazole (E) (Scheme 3).

The plausible mechanism for the synthesis of 1,2,4,5-tetra substituted imidazoles [33] with the copper nano ferrite in the reaction may be shown in Scheme 4. Ammonium acetate is the source of ammonia molecule. Aldehyde and 1, 2-diketone are first activated by ferrite nano particles $\left(\mathrm{Fe}^{3+}\right)$. Then, aldehyde, ammonia and Substituted amine undergo reaction to form germinal di-amino intermediate (A). Further reaction of activated 1,2-diketone (B) with germinal di amino intermediate (A) by the loss of water molecule to form an intermediate (C) on cyclization gives intermediate (D) which on loss of one more water molecule gives tetra substituted imidazole (E) (Scheme 4).

\subsection{Optimization of Reaction Conditions}

Since few years as a part of our ongoing investigation in developing an efficient and green synthesis for synthesis of poly substituted imidazoles, here we report an efficient method for the synthesis of poly substituted

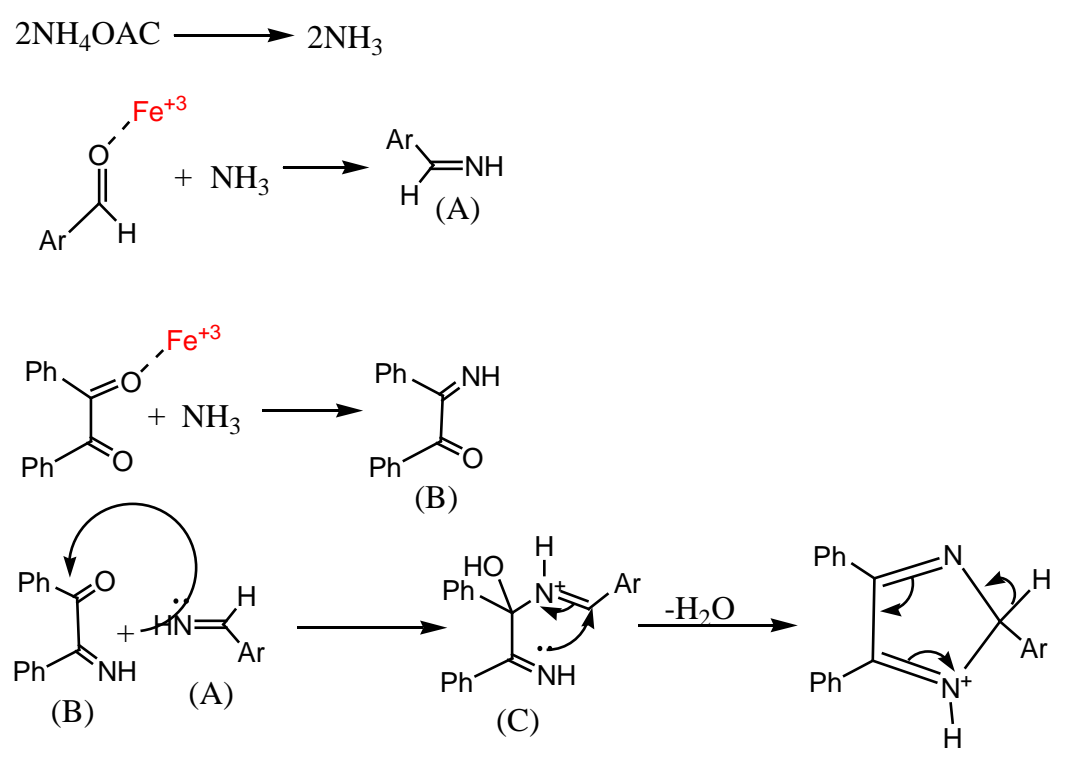

(D)<smiles></smiles>

(E) 
imidazoles by cyclo condensation of benzil, aromatic aldehydes, ammonium acetate and substituted amines in ethanol in presence of copper nano ferrite under ultrasound irradiation.

To evaluate the effect of ultrasound on Scheme 1 initially reaction was performed with same conditions in absence of ultrasound irradiation, which results in low to moderate yields of formation of products and but under ultra sonication results good yields of products using the optimized reaction conditions. The formation of moderate yields in absence of sonication is due to effect of copper nano ferrite. The results are tabulated in Table 1.

This process was demonstrated by taking wide range of substituted aromatic aldehydes to synthesize the corresponding products in good yields. Aromatic aldehydes bearing electron releasing groups leads some better results than electron withdrawing groups. This methodology was compared with the other ultrasonic methods which are performed in presence of different catalyst at different reaction temperatures. The results are tabulated in Table 2.
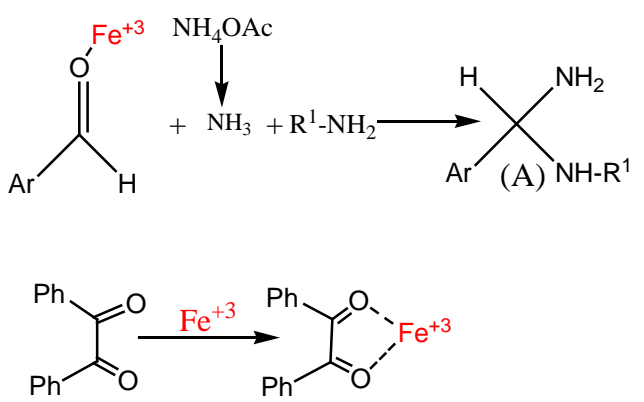

(B)

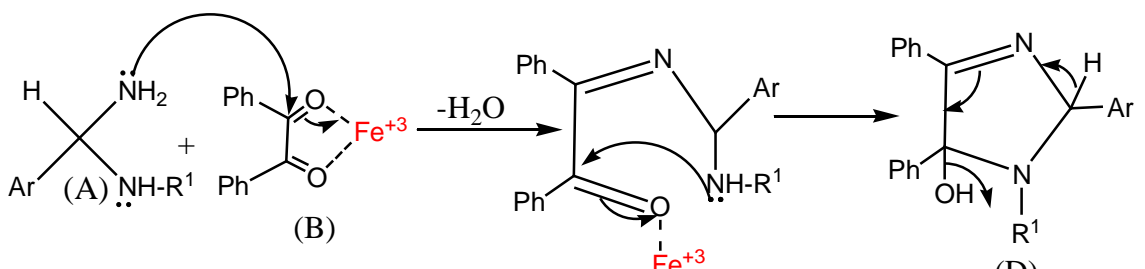

(C)

(D)<smiles></smiles>

(E)

Scheme 4. Plausible mechanism for the formation of 2,4,5-tri substituted Imidazoles.

Table 1. Effect of ultrasound on Scheme 1 (compound 4a).

\begin{tabular}{cccc}
\hline S. No. & Condition & Time (min) & Yield (\%) $^{(\mathrm{a})}$ \\
\hline 1 & No sonication & 30 & 45 \\
2 & No sonication & 45 & 55 \\
3 & Sonication at R.T. & 10 & 60 \\
4 & Sonication at R.T. & 20 & 80 \\
5 & Sonication at R.T. & 30 & 96
\end{tabular}

(a) Reaction conditions: Benzil (10 mmol), m-bromo benzaldehyde (10 mmol), Ammonium acetate (10 mmol), Copper ferrite (2 mmol) and Ethanol $(5.0 \mathrm{~mL})$. 
Table 2. One-pot Synthesis of various 2,4,5-tri substituted imidazole derivatives under ultrasound irradiation.

\begin{tabular}{|c|c|c|c|c|c|c|}
\hline S. No. & U.S./ Catalyst & $\mathrm{R}$ & Temp $\left({ }^{\circ} \mathrm{C}\right)$ & Time (min) & Yield (\%) & M.P. $\left({ }^{\circ} \mathrm{C}\right)_{\text {rep }} /$ M.P. $\left({ }^{\circ} \mathrm{C}\right)_{\text {lit }}$ \\
\hline 1 & U.S./SA-MNPs [22] & $\mathrm{m}-\mathrm{Br}$ & 40 & 30 & 95 & 302-304/301-303 [22] \\
\hline 2 & U.S./[EMIM]OAc [23] & $\mathrm{m}-\mathrm{Br}$ & - & - & - & Not reported \\
\hline 3 & U.S./CuFe ${ }_{2} \mathrm{O}_{4}$ & $\mathrm{~m}-\mathrm{Br}$ & R.T. & 30 & $96(4 a)$ & 300-302/301-303 [22] \\
\hline 4 & U.S./SA-MNPs [22] & $\mathrm{p}-\mathrm{F}$ & - & - & - & Not reported \\
\hline 5 & U.S./[EMIM]OAc [23] & $\mathrm{p}-\mathrm{F}$ & - & - & - & Not reported \\
\hline 6 & U.S. $/ \mathrm{CuFe}_{2} \mathrm{O}_{4}$ & $\mathrm{p}-\mathrm{F}$ & R.T. & 25 & $92(4 b)$ & $188-190 / 190^{(19)}$ \\
\hline 7 & U.S./SA-MNPs [22] & $\mathrm{p}-\mathrm{CH}_{3}$ & 40 & 40 & 92 & 187-189/186-188 [22] \\
\hline 8 & U.S./[EMIM]OAc [23] & $\mathrm{p}-\mathrm{CH}_{3}$ & - & - & - & Not reported \\
\hline 9 & U.S./CuFe ${ }_{2} \mathrm{O}_{4}$ & $\mathrm{p}-\mathrm{CH}_{3}$ & R.T. & 30 & $94(4 \mathrm{e})$ & 184-186/186-188 [22] \\
\hline 10 & U.S./SA-MNPs [22] & $\mathrm{m}-\mathrm{NO}_{2}$ & 40 & 30 & 92 & 268-270/269-271 [22] \\
\hline 11 & U.S./[EMIM]OAc [23] & $\mathrm{m}-\mathrm{NO}_{2}$ & R.T. & 90 & 87 & 317-319/313-315 [23] \\
\hline 12 & U.S./CuFe ${ }_{2} \mathrm{O}_{4}$ & $\mathrm{~m}-\mathrm{NO}_{2}$ & R.T. & 30 & $88(4 c)$ & $310-312 / 313-315$ [23] \\
\hline 13 & U.S./SA-MNPs [22] & $\mathrm{p}-\mathrm{NO}_{2}$ & - & - & - & Not reported \\
\hline 14 & U.S./[EMIM]OAc [23] & $\mathrm{p}-\mathrm{NO}_{2}$ & R.T. & 70 & 70 & 239-242/235-238 [23] \\
\hline 15 & U.S./CuFe ${ }_{2} \mathrm{O}_{4}$ & $\mathrm{p}-\mathrm{NO}_{2}$ & R.T. & 30 & $86(4 d)$ & 232-234/235-238 [23] \\
\hline
\end{tabular}

S. Nos. 1-9 contain electron releasing groups where as SNos.10-15 contain electron withdrawing groups on the aldehyde

U.S. = Ultra Sonication; SA-MNPs = Sulfamic acid-functionalized magnetic nano particles; [EMIM] OAc = 1-ethyl-3-methylimidazole acetate; $\mathrm{CuFe}_{2} \mathrm{O}_{4}=$ copper ferrite nano particles (present work). Reaction conditions: Benzil (10 mmol), Aromatic aldehyde (10 mmol), Ammonium acetate (10 mmol), Copper ferrite $(2 \mathrm{mmol})$ and Ethanol $(5.0 \mathrm{~mL})$. As mentioned above some of the tri substituted imidazole products with the respective catalysts were not reported with the mentioned aromatic aldehyde.

In extension to our work here we report an efficient method for the synthesis of tetra substituted imidazoles by cyclo condensation of benzil, aromatic aldehydes, ammonium acetate and substituted amines in ethanol in presence of copper nano ferrite under ultrasound irradiation. The results are tabulated in Table 3.

In order to examine the effect of catalyst concentration on Scheme 2 (product 9c) we perform the reaction with various concentrations of the catalyst in ethanol at $40^{\circ} \mathrm{C}$. The use of $2 \mathrm{mmol}$ and above concentrations of the catalyst gave good yields of the desired products. The results are tabulated in Table 4.

In order to examine the effect of solvent on Scheme 2 we perform the reaction in presence of various solvents including ethanol, $\mathrm{CH}_{3} \mathrm{CN}, \mathrm{CH}_{2} \mathrm{Cl}_{2}$ and water at different time at $40^{\circ} \mathrm{C}$. The use of ethanol as solvent afforded good yields of the desired products. The results are tabulated in Table 5.

\subsection{Recycling of the Catalyst}

Catalyst reusability is of major concern in heterogeneous catalysis. The recovery and reusability of the catalyst was investigated in this reaction with benzaldehyde (4a). Catalyst recycling was achieved by fixing the catalyst magnetically at the bottom of the flask with a strong magnet, after which the solution was taken off with a pipette and concentrated in rotary evaporator then the solid washed twice with chloroform. The fresh substrate dissolved in the same solvent was introduced into the flask, allowing the reaction to proceed for the next run.The catalyst was consecutively reused five times without any noticeable loss of its catalytic activity. These catalysts are highly magnetic and their saturation magnetization values [46] are much higher than other reported magnetic catalysts. Therefore, they could be easily and almost completely separated by an external magnet which is of a great advantage for a heterogeneous catalyst. 
Table 3. One-pot Synthesis of various 1,2,4,5-tetra substituted imidazole derivatives under ultrasound irradiation.

\begin{tabular}{|c|c|c|c|c|c|c|c|}
\hline S. No. & U.S./Reflux Under Catalyst & $\mathrm{R}$ & $\mathrm{R}^{1}$ & Temp $\left({ }^{\circ} \mathrm{C}\right)$ & Time (min) & Yield (\%) & M.P. $\left({ }^{\circ} \mathrm{C}\right)_{\mathrm{rep}} / \mathrm{M} . P .\left({ }^{\circ} \mathrm{C}\right)_{\mathrm{lit}}$ \\
\hline 1 & $\mathrm{HClO}_{4}-\mathrm{SiO}_{2} /$ reflux [29] & $\mathrm{H}$ & $\mathrm{C}_{6} \mathrm{H}_{5}$ & 140 & 10 & 91 & 216 [29] \\
\hline 2 & $\mathrm{MgAl}_{2} \mathrm{O}_{4} /$ U.S. [33] & $\mathrm{H}$ & $\mathrm{C}_{6} \mathrm{H}_{5}$ & 60 & 15 & 91 & $216-218$ [33] \\
\hline 3 & $\mathrm{CuFe}_{2} \mathrm{O}_{4} /$ U.S. & $\mathrm{H}$ & $\mathrm{C}_{6} \mathrm{H}_{5}$ & 40 & 15 & 92(9a) & 214-216/216 [29] \\
\hline 4 & $\mathrm{HClO}_{4}-\mathrm{SiO}_{2} /$ reflux [29] & $\mathrm{p}-\mathrm{OCH}_{3}$ & $\mathrm{C}_{6} \mathrm{H}_{5}$ & - & - & - & Not reported \\
\hline 5 & $\mathrm{MgAl}_{2} \mathrm{O}_{4} /$ U.S. [33] & $\mathrm{p}-\mathrm{OCH}_{3}$ & $\mathrm{C}_{6} \mathrm{H}_{5}$ & 60 & 18 & 93 & 253-254 [33] \\
\hline 6 & $\mathrm{CuFe}_{2} \mathrm{O}_{4} /$ U.S. & $\mathrm{p}-\mathrm{OCH}_{3}$ & $\mathrm{C}_{6} \mathrm{H}_{5}$ & 40 & 15 & $94(9 b)$ & 254-256/253-254 [33] \\
\hline 7 & $\mathrm{HClO}_{4}-\mathrm{SiO}_{2} /$ reflux [29] & $\mathrm{H}$ & $\mathrm{C}_{6} \mathrm{H}_{5}-\mathrm{CH}_{2}$ & 140 & 6 & 96 & $165-167$ [29] \\
\hline 8 & $\mathrm{MgAl}_{2} \mathrm{O}_{4} /$ U.S. [33] & $\mathrm{H}$ & $\mathrm{C}_{6} \mathrm{H}_{5}-\mathrm{CH}_{2}$ & - & - & - & Not reported \\
\hline 9 & $\mathrm{CuFe}_{2} \mathrm{O}_{4} /$ U.S. & $\mathrm{H}$ & $\mathrm{C}_{6} \mathrm{H}_{5}-\mathrm{CH}_{2}$ & 40 & 10 & 95(9c) & 166-168/165-167 [29] \\
\hline 10 & $\mathrm{HClO}_{4}-\mathrm{SiO}_{2} /$ reflux [29] & $\mathrm{p}-\mathrm{OCH}_{3}$ & $\mathrm{C}_{6} \mathrm{H}_{5}-\mathrm{CH}_{2}$ & 140 & 10 & 96 & $157-160[29]$ \\
\hline 11 & $\mathrm{MgAl}_{2} \mathrm{O}_{4} /$ U.S. [33] & $\mathrm{p}-\mathrm{OCH}_{3}$ & $\mathrm{C}_{6} \mathrm{H}_{5}-\mathrm{CH}_{2}$ & - & - & - & Not reported \\
\hline 12 & $\mathrm{CuFe}_{2} \mathrm{O}_{4} / \mathrm{U} . \mathrm{S}$. & $\mathrm{p}-\mathrm{OCH}_{3}$ & $\mathrm{C}_{6} \mathrm{H}_{5}-\mathrm{CH}_{2}$ & 40 & 15 & 94(9d) & $158-160 / 157-160$ [29] \\
\hline 13 & $\mathrm{HClO}_{4}-\mathrm{SiO}_{2} /$ reflux [29] & $\mathrm{p}-\mathrm{CH}_{3}$ & $\mathrm{C}_{6} \mathrm{H}_{5}-\mathrm{CH}_{2}$ & 140 & 6 & 90 & $165-168$ [29] \\
\hline 14 & $\mathrm{MgAl}_{2} \mathrm{O}_{4} /$ U.S. [33] & $\mathrm{p}-\mathrm{CH}_{3}$ & $\mathrm{C}_{6} \mathrm{H}_{5}-\mathrm{CH}_{2}$ & - & - & - & Not reported \\
\hline 15 & $\mathrm{CuFe}_{2} \mathrm{O}_{4} / \mathrm{U} . \mathrm{S}$. & $\mathrm{p}-\mathrm{CH}_{3}$ & $\mathrm{C}_{6} \mathrm{H}_{5}-\mathrm{CH}_{2}$ & 40 & 20 & 96(9e) & 168-170/165-168 [29] \\
\hline 16 & $\mathrm{HClO}_{4}-\mathrm{SiO}_{2} /$ reflux [29] & $\mathrm{p}-\mathrm{Cl}$ & $\mathrm{C}_{6} \mathrm{H}_{5}-\mathrm{CH}_{2}$ & 140 & 8 & 94 & $162-164 / 162-165$ [29] \\
\hline 17 & $\mathrm{MgAl}_{2} \mathrm{O}_{4} /$ U.S. [33] & $\mathrm{p}-\mathrm{Cl}$ & $\mathrm{C}_{6} \mathrm{H}_{5}-\mathrm{CH}_{2}$ & - & - & - & Not reported \\
\hline 18 & $\mathrm{CuFe}_{2} \mathrm{O}_{4} / \mathrm{U} . \mathrm{S}$. & $\mathrm{p}-\mathrm{Cl}$ & $\mathrm{C}_{6} \mathrm{H}_{5}-\mathrm{CH}_{2}$ & 40 & 15 & 92(9f) & $160-162 / 162-163$ [26] \\
\hline 19 & $\mathrm{HClO}_{4}-\mathrm{SiO}_{2} /$ reflux [29] & $\mathrm{p}-\mathrm{Cl}$ & Cyclohexyl & - & - & - & Not reported \\
\hline 20 & $\mathrm{MgAl}_{2} \mathrm{O}_{4} /$ U.S. [33] & $\mathrm{p}-\mathrm{Cl}$ & Cyclohexyl & - & - & - & Not reported \\
\hline 21 & $\mathrm{CuFe}_{2} \mathrm{O}_{4} /$ U.S. & $\mathrm{p}-\mathrm{Cl}$ & Cyclohexyl & 40 & 20 & $90(9 g)$ & $152-154 /-$ \\
\hline 22 & $\mathrm{HClO}_{4}-\mathrm{SiO}_{2} /$ reflux [29] & $\mathrm{p}-\mathrm{CH}_{3}$ & Cyclohexyl & 140 & 18 & 90 & 164 [29] \\
\hline 23 & $\mathrm{MgAl}_{2} \mathrm{O}_{4} /$ U.S. [33] & $\mathrm{p}-\mathrm{CH}_{3}$ & Cyclohexyl & - & - & - & Not reported \\
\hline 24 & $\mathrm{CuFe}_{2} \mathrm{O}_{4} /$ U.S. & $\mathrm{p}-\mathrm{CH}_{3}$ & Cyclohexyl & 40 & 20 & $92(9 h)$ & $162-164 / 164$ [29] \\
\hline
\end{tabular}

U.S. = Ultra Sonication, $\mathrm{CuFe}_{2} \mathrm{O}_{4}=$ copper ferrite nano particles (present work). Reaction conditions: Benzil (10 mmol), Aromatic aldehyde (10 mmol), Ammonium acetate $(10 \mathrm{mmol})$, substituted amine $(10 \mathrm{mmol})$, Copper ferrite $(2 \mathrm{mmol})$ and Ethanol $(5.0 \mathrm{~mL})$. As mentioned above some of the tri substituted imidazole products with the respective catalysts were not reported with the mentioned aromatic aldehyde.

Table 4. Effect of catalyst concentration on the synthesis of 1,2,4,5-tetra substituted imidazoles ${ }^{[a]}$.

\begin{tabular}{ccc}
\hline S. No. & $\mathrm{CuFe}_{2} \mathrm{O}_{4}$ Concentration mmol & Yield (\%) \\
\hline 1 & 5 & 95 \\
2 & 2 & 85 \\
3 & 1 & 70 \\
4
\end{tabular}

[a] Reaction conditions for Product 9c: Benzil (10 mmol), Benzaldehyde (10 mmol), Ammonium acetate (10 mmol), Benzyl amine (10 mmol), Etha$\operatorname{nol}(5.0 \mathrm{~mL}), 40^{\circ} \mathrm{C}$. 
Table 5. Effect of solvent on the synthesis of 1,2,4,5-tetra substituted imidazoles ${ }^{[\mathrm{b}]}$.

\begin{tabular}{ccc}
\hline S. No. & Solvent & Yield (\%) \\
\hline 1 & Ethanol & 95 \\
2 & $\mathrm{CH}_{3} \mathrm{CN}$ & 65 \\
3 & $\mathrm{CH}_{2} \mathrm{Cl}_{2}$ & 35 \\
4 & Water & Trace \\
\hline
\end{tabular}

[b] Reaction conditions: Benzil (10 mmol), Benzaldehyde (10 mmol), Ammonium acetate (10 mmol), Benzyl amine (10 mmol), Copper ferrite (2 $\mathrm{mmol})$, Ethanol $(5.0 \mathrm{~mL}), 40^{\circ} \mathrm{C}$.

\section{Conclusion}

In conclusion, we have reported an efficient, convenient and environmentally benign ultrasound assisted one-pot greener synthesis of poly substituted imidazole derivatives using nano copper ferrite. The notable features offered by this methodology are mild reaction conditions, simple procedure, cleaner reactions and good yields of products.

\section{Acknowledgements}

The authors wish to thank the UGC for the all the facilities received through the Major Research Project No. F. 41-371/2012 (SR) to Paul Douglas Sanasi and CSIR-SRF to Ravi Kumar Majji, UGC-SRF to Swathi Bandaru.

\section{References}

[1] Ugi, I. (2001) Recent Progress in the Chemistry of Multi Component Reactions. Pure and Applied Chemistry, 73, 187191.

[2] Domling, A. (2006) Recent Developments in Isocyanide Based Multicomponent Reactions in Applied Chemistry. Chemical Reviews, 106, 17-89.

[3] Lambardino, J.G. and Wiseman, E.H. (1974) Preparation and Anti-Inflammatory Activity of Some Non-Acidic Trisubstituted Imidazoles. Journal of Medicinal Chemistry, 17, 1182-1188.

[4] Puratchikody, A. and Doble, M. (2007) Antinociceptive and Anti-Inflammatory Activities and QSAR Studies on 2Substituted-4, 5 Diphenyl, 1-H imidazole. Bioorganic \& Medicinal Chemistry Letters, 15, 1083-1090.

[5] Maier, T., Schmierer, R., Bauer, K., Bieringer, H., Buerstell, H. and Sachse, B. (1989) US Patent 4820335. Chemical Abstracts, 11, 19494.

[6] Heeres, J., Backx, L.J.J., Mostmans, J.H. and Van Custem, J. (1979) Antimycotic Imidazoles: Part 4, Synthesis and Antifungal Activity of Ketoconazole, a New Potent Orally Active Broad Spectrum Antifungal Agent. Journal of Medicinal Chemistry, 22, 1003-1005.

[7] Schmierer, R., Mildenberger, H. and Buerstell, H. (1988) German Patent 361464. Chemical Abstracts, 108, 37838.

[8] Lombardino, J.G., and Wiseman, E.H. (1974) Preparation and Anti-Inflammatory Activity of Some Non-Acidic Trisubstituted Imidazoles. Journal of Medicinal Chemistry, 17, 1182-1188.

[9] Phillips, A.R., White, H.L. and Rosen, S. (1982) European Patent Application EP 58 8901, 1982; Chemical Abstracts, 98, 53894z.

[10] Wang, L., Woods, K.W., Li, Q., Barr, K.J., McCroskey, R.W., Hannick, S.M., Gherke, L., Credo, R.B., Hui, Y.H., Marsh, K., Warner, R., Lee, J.Y., Zielinsky, Y., Frost, D., Rosenberg, S.H. and Sham, H.L. (2002) Potent-Orally Active-Based Heterocycle Combretastatine A-4 Analogues: Synthesis, Structure-Activity Relationship, Pharmacokinetics and in Vivo Antitumor Activity Evalution. Journal of Medicinal Chemistry, 45, 1697-1711.

[11] Lantos, I., Zhang, W.Y., Shiu, X. and Eggleston, D.S. (1993) Synthesis of Imidazole via Hetero-Cope Rearrangements. The Journal of Organic Chemistry, 58, 7092-7095. http://dx.doi.org/10.1021/jo00077a033

[12] Zhang, C., Moran, E.J., Woiwade, T.F., Short, K.M. and Mjalli, A.M. (1996) Synthesis of Tetra Substituted Imidazoles via $\alpha$-( $N$-acyl- $N$-alkylamino)- $\beta$-ketoamides on Wang Resin. Tetrahedron Letters, 37, 751-754. http://dx.doi.org/10.1016/0040-4039(95)02310-0

[13] Rajaguru, K., Suresh, R., Mariappan, A., Muthusubramanian, S. and Bhuvanesh, N. (2014) Erbium Triflate Promoted Multicomponent Synthesis of Highly Substituted Imidazoles. Organic Letters, 16, 744-747. 
http://dx.doi.org/10.1021/ol403456b

[14] (a) Radziszewski, B. (1882) Ueber die Constitution des Lophins und verwandter Verbindungen. Chemische Berichte, 15, 1493-1496. http://dx.doi.org/10.1002/cber.18820150207

(b) Japp, F. and Robinson, H. (1882) Constitution des Lophins und des Amarins. Chemische Berichte, 15, 1268-1270. http://dx.doi.org/10.1002/cber.188201501272

[15] Heravi, M.M., Bekhtiari, K., Oskooie, H.A. and Taheri, S. (2007) Synthesis of 2,4,5-Triaryl Imidazoles Catalyzed by $\mathrm{NiCl}_{2} \cdot 6 \mathrm{H}_{2} \mathrm{O}$ under Heterogeneous System. Journal of Molecular Catalysis A: Chemical, 263, 279-281. http://dx.doi.org/10.1016/j.molcata.2006.08.070

[16] Bhosale, S.V., Kalyankar, M.B., Nalage, S.V., Bhosale, D.S., Pandhare, S.L., Kotbagi, T.V., Umbarkar, S.B. and Dongare, M.K. (2011) One-Pot Synthesis of 2,4,5-Tri Substituted Imidazoles Using $\mathrm{MoO}_{3} / \mathrm{SiO}_{2}$, an Efficient and Recyclable Catalyst. Synthetic Communications, 41, 762-769. http://dx.doi.org/10.1080/00397911003644415

[17] Wang, L. and Cai, C. (2009) Polymer Supported Zinc Chloride: A Highly Active and Reusable Heterogeneous Catalyst for One-Pot Synthesis of 2,4,5-Tri Substituted Imidazoles. Monatshefte für Chemie, 140, 541-546. http://dx.doi.org/10.1007/s00706-008-0086-2

[18] Hojati, S.F., Nezhadhoseiny, S.A. and Beykzadeh, Z. (2013) Trichloro Isocyanuric Acid-Catalysed One-Pot Synthesis of 2,4,5-Tri Substituted Imidazoles. Monatshefte für Chemie, 144, 387-390. http://dx.doi.org/10.1007/s00706-012-0830-5

[19] Shelke, K., Kakade, G., Shingate, B. and Shingare, M. (2008) Microwave Induced One-Pot Synthesis of 2,4,5-Tri Aryl Imidazoles Using Glyoxylic Acid as a Catalyst under Solvent Free Condition. Rasayan Journal of Chemistry, 3, 489494.

[20] Safari, J., Khalili, S.D., Banitaba, S.H. and Dehghani, H. (2011) Zinc(II) [Tetra(4-methylphenyl)] Porphyrine: A Novel and Reusable Catalyst for Efficient Synthesis of 2,4,5-Tri Substituted Imidazoles under Ultra Sound Irradiation. Journal of the Korean Chemical Society, 55, 787-793. http://dx.doi.org/10.5012/jkcs.2011.55.5.787

[21] Safari, J. and Zarnegar, Z. (2012) Magnetic $\mathrm{Fe}_{3} \mathrm{O}_{4}$ Nanoparticles as a Highly Efficient Catalyst for Synthesis of Imidazoles under Ultrasound Irradiation. Iranian Journal of Catalysis, 2, 121-128. http://www.sid.ir/en/VEWSSID/J pdf/1036220120305.pdf

[22] Safari, J. and Zarnegar, Z. (2013) A Highly Efficient Magnetic Solid Acid Catalyst for Synthesis of 2,4,5-Tri Substituted Imidazoles under Ultra Sound Irradiation. Ultrasonics Sonochemistry, 20, 740-746. http://dx.doi.org/10.1016/j.ultsonch.2012.10.004

[23] Zang, H.J., Su, Q.H., Mo, Y.M., Cheng, B.-W. and Jun, S. (2010) Ionic Liquid [EMIM]OAC under Ultra Sonic Irradiation towards First Synthesis of Tri Substituted Imidazoles. Ultrasonics Sonochemistry, 17, 749-751. http://dx.doi.org/10.1016/j.ultsonch.2010.01.015

[24] Ahmad, R.K. (2008) Ultra Sound Promoted Greener Synthesis of 2,4,5-Tri Substituted Imidazoles Catalysed by $\mathrm{Zr}(\mathrm{acac})_{4}$ under Ambient Conditions. Ultrasonics Sonochemistry, 15, 659-664. http://dx.doi.org/10.1016/j.ultsonch.2007.12.005

[25] Shelke, K.F., Sapkal, S.B. and Shingare, M.S. (2009) Ultra Sound-Assisted One-Pot Synthesis of 2,4,5-Tri Aryl Imidazoles Catalyzed by Ceric(IV) Ammonium Nitrate in Aqueous Media. Chinese Chemical Letters, 20, 283-287. http://dx.doi.org/10.1016/j.cclet.2008.11.033

[26] Mirjalili, B.F., Bamoniri, A.H. and Zamani, L. (2012) One-Pot Synthesis of 1,2,4,5-Tetra Substituted Imidazoles Promoted by Nano $\mathrm{TiCl}_{4} \cdot \mathrm{SiO}_{2}$. Scientia Iranica, 19, 565-568. http://dx.doi.org/10.1016/j.scient.2011.12.013

[27] Majid, M.H., Fatemeh, D. and Masoumeh, H. (2008) Highly Efficient, Four Component One-Pot Synthesis of Tetra Substituted Imidazoles Using a Catalytic Amount of $\mathrm{FeCl}_{3} \cdot 6 \mathrm{H}_{2} \mathrm{O}$. Monatshefte für Chemie, 139, 31-33. http://dx.doi.org/10.1007/s00706-007-0736-9

[28] Sadeghi, B., Mirjalili, B.B.F. and Hashemi, M.M. (2008) $\mathrm{BF}_{3} \cdot \mathrm{SiO}_{2}$ : An Efficient Reagent System for the One-Pot Synthesis of 1,2,4,5-Tetra Substituted Imidazoles. Tetrahedron Letters, 49, 2575-2577. http://dx.doi.org/10.1016/j.tetlet.2008.02.100

[29] Kantevari, S., Vuppalapati, S.V.N., Biradar, D.O. and Nagarapu, L. (2007) Highly Efficient, One-Pot, Solvent Free One-Pot Synthesis of Tetra Substituted Imidazoles Using $\mathrm{HClO}_{4}-\mathrm{SiO}_{2}$ as Novel Heterogeneous Catalyst. Journal of Molecular Catalysis A: Chemical, 266, 109-113. http://dx.doi.org/10.1016/j.molcata.2006.10.048

[30] Heravi, M.M., Drikvand, F.F. and Bamoharram, F. (2007) Highly Efficient Four Component One-Pot Synthesis of Tetra Substituted Imidazoles Using Keggin-Type Hetero Poly Acids as Green and Reusable Catalyst. Journal of Molecular Catalysis A: Chemical, 263, 112-114. http://dx.doi.org/10.1016/j.molcata.2006.08.048

[31] Nagarapu, L., Apuri, S. and Kantevari, S. (2007) Pottasium Dodecatungstocobaltate Trihydrate $\left(\mathrm{K}_{5} \mathrm{CoW}_{12} \mathrm{O}_{40} \cdot 3 \mathrm{H}_{2} \mathrm{O}\right)$; a Mild and Efficient Reusable Catalyst for the One-Pot Synthesis of 1,2,4,5-Tetra Substituted Imidazoles under Conventional Heating and Microwave Irradiation. Journal of Molecular Catalysis A: Chemical, 266, 104-108. 
http://dx.doi.org/10.1016/j.molcata.2006.10.056

[32] Saeed, B. and Arabanian, A. (2000) One-Pot Synthesis of Tetra Substituted Imidazoles Catalysed by Zeolite HY and Silicagel under Microwave Irradiation. Green Chemistry, 2, 274-276. http://dx.doi.org/10.1039/b0062010

[33] Safari, J., Gandomi-Ravandi, S. and Akbari, Z. (2013) Sonochemical Synthesis of 1,2,4,5-Tetra Substituted Imidazoles Using Nano Crystalline $\mathrm{MgAl}_{2} \mathrm{O}_{4}$ as an Effective Catalyst. Journal of Advanced Research, 4, 509-514. http://dx.doi.org/10.1016/j.jare.2012.09.001

[34] Kidwai, M., Mothsra, P., Bansal, V., Somvanshi, R.K., Ethayathulla, A.S., Dey, S. and Singh, T.P. (2007) One-Pot Synthesis of Highly Substituted Imidazoles Using Molecular Iodine: A Versatile Catalyst. Journal of Molecular Catalysis A: Chemical, 265, 177-182. http://dx.doi.org/10.1016/j.molcata.2006.10.009

[35] Karimi-Jaberi, Z. and Barekat, M. (2010) One-Pot Synthesis of Tri and Tetra Substituted Imidazoles Using Sodium Dihydrogen Phosphate under Solvent-Free Conditions. Chinese Chemical Letters, 21, 1183-1186. http://dx.doi.org/10.1016/j.cclet.2010.06.012

[36] Sharma, R.K., Sharma, C. and Prerna (2012) An Efficient and One Pot Synthesis Poly Substituted Imidazoles by BiCl . Indian Journal of Chemistry, 51B, 1489-1493.

[37] Narayana Murthy, S., Madhav, B. and Nageswar, Y.V.D. (2010) DABCO as a Mild and Efficient Catalytic System for the Synthesis of Highly Substituted Imidazoles via Multicomponent Condensation Strategy. Tetrahedron Letters, 51, 5252-5257. http://dx.doi.org/10.1016/j.tetlet.2010.07.128

[38] Ziarani, G.M., Badiei, A., Lashgari, N. and Farahani, Z. (2013) Efficient One-Pot Synthesis of 2,4,5-Tri Substituted and 1,2,4,5-Tetra Substituted Imidazoles Using SBA-Pr- $\mathrm{SO}_{3} \mathrm{H}$ as a Green Nano Catalyst. Journal of Saudi Chemical Society. (in press)

[39] Samai, S., Nandi, G.C., Singh, P. and Singh, M.S. (2009) L-Proline: An Efficient Catalyst for the One-Pot Synthesis of 2,4,5-Tri Substituted and 1,2,4,5-Tetra Substituted Imidazoles. Tetrahedron, 65, 10155-10161. http://dx.doi.org/10.1016/j.tet.2009.10.019

[40] Teimouri, A. and Chermahini, A.N. (2011) An Efficient and One-Pot Synthesis of 2,4,5-Tri Substituted and 1,2,4,5Tetra Substituted Imidazoles Catalysed via Solid Acid Nano Catalyst. Journal of Molecular Catalysis A: Chemical, 346, 39-45. http://dx.doi.org/10.1016/j.molcata.2011.06.007

[41] Sharma, S.D., Hazarika, P. and Konwar, D. (2008) An Efficient and One-Pot Synthesis of 2,4,5-Tri Substituted and 1,2,4,5-Tetra Substituted Imidazoles Catalysed by $\mathrm{InCl}_{3} \cdot 3 \mathrm{H}_{2} \mathrm{O}$. Tetrahedron Letters, 49, 2216-2220. http://dx.doi.org/10.1016/j.tetlet.2008.02.053

[42] Karimi, A.R., Alimohammadi, Z. and Mostafa, M.A. (2010) Well-Dawson Heteropolyacid Supported on Silica: A Highly Efficient Catalyst for Synthesis of 2,4,5-Tri Substituted and 1,2,4,5-Tetra Substituted Imidazoles. Molecular Diversity, 14, 635-641. http://dx.doi.org/10.1007/s11030-009-9197-X

[43] Kanazawa, C., Kamijo, S. and Yamamoto, Y. (2006) Synthesis of Imidazoles through Copper Catalysed Cross-Cycloaddition between Two Different Isocyanides. Journal of the American Chemical Society, 128, 10662-10663. http://dx.doi.org/10.1021/ja0617439

[44] Li, J.H. and Neuville, L. (2013) Copper-Catalyzed Oxidative Diamination of Terminal Alkynes by Amidines: Synthesis of 1,2,4-Tri Substituted Imidazoles. Organic Letters, 15, 1752-1755. http://dx.doi.org/10.1021/ol400560m

[45] Tang, D., Wu, P., Liu, X., Chen, Y.-X., Guo, S.-B., Chen, W.-L., Li, J.-G. and Chen, B.-H. (2013) Synthesis of Multi Substituted Imidazoles via Copper Catalysed [3+2] Cycloadditions. The Journal of Organic Chemistry, 78, $2746-2750$. http://dx.doi.org/10.1021/jo302555z

[46] Paul Douglas, S., Santhipriya, D., Ramesh, Y., Ravi Kumar, M., Swathi, B. and Jaya Rao, K. (2014) Nano Copper and Cobalt Ferrites as Heterogeneous Catalysts for the One-Pot Synthesis of 2,4,5-Tri Substituted Imidazoles. Journal of Chemical Sciences, 126, 1715-1720. http://dx.doi.org/10.1007/s12039-014-0729-2

[47] Ganesh Babu, S. and Karvembu, R. (2013) Copper Based Nano Particles-Catalysed Organic Transformations. Catalysis Surveys from Asia, 17, 156-176. http://dx.doi.org/10.1007/s10563-013-9159-2

[48] Lakshmi Kantam, M., Yadav, J., Laha, S., Srinivas, P., Sreedhar, B. and Figueras, F.J. (2009) Asymmetric Hydrosilylation of Ketones Catalysed by Magnetically Recoverable and Reusable Copper Ferrite Nanoparticles. The Journal of Organic Chemistry, 74, 4608-4611.

[49] Swapna, K., Narayana Murthy, S., Tarani Jyothi, M. and Venkata Durga Nageswar, Y. (2011) Nano Copper Ferrite as Magnetically Separable and Reusable Catalyst for the Synthesis of Diaryl/Alkyl Sulfides via Cross-Coupling Process under Ligand Free Conditions. Organic \& Biomolecular Chemistry, 9, 5989-5996. http://dx.doi.org/10.1039/c1ob05597fc

[50] Daoshan, Y., Xia, Z., Wei, W., Min, J., Ning, Z., Dandan, R., Jinmao, Y. and Hua, W. (2014) Magnetic Copper Ferrite Nano Particles: An Inexpensive, Efficient, Recyclable Catalyst for the Synthesis of Substituted Benzoxazoles via Ullmann Type Coupling under Ligand-Free Conditions. Synlett, 25, 729-735. http://dx.doi.org/10.1055/s-0033-1340599 
[51] Swapna, K., Narayana Murthy, S. and Venkata Durga Nageswar, Y. (2011) Magnetically Separable and Reusable Copper Ferrite Nano Particles for Cross-Coupling of Aryl Halides with Diphenyl Selinides. European Journal of Organic Chemistry, 2011, 1940-1946. http://dx.doi.org/10.1002/ejoc.201001639

[52] Ayoob, B., Ghaffar, H. and Ramin, G. (2013) Copper Ferrite Nano Particles: An Efficient and Reusable Nano Catalyst for a Green One-Pot Three Component Synthesis of Spiro Oxindoles in Water. ACS Combinatorial Science, 15, 530534. http://dx.doi.org/10.1021/c0400057h

[53] Anil Kumar, B.S.P., Harsha Vardhan Reddy, K., Madhav, B., Ramesh, K. and Nageswar, Y.V.D. (2012) Magnetically Separable $\mathrm{CuFe}_{2} \mathrm{O}_{4}$ Nano Particles Catalysed Multicomponent Synthesis of 1,4-disubstituted 1,2,3-Triazoles in Tap Water. Tetrahedron Letters, 53, 4595-4599. http://dx.doi.org/10.1016/j.tetlet.2012.06.077

[54] (a) Li, J.T., Sun, M.X. and Yin, Y. (2010) Ultra Sound Promoted Efficient Method for the Cleavage of 3-Aryl, 2,3Epoxyl, 1-Phenyl,1-Propanone with Indole. Ultrasonics Sonochemistry, 17, 359-362. http://dx.doi.org/10.1016/j.ultsonch.2009.09.004

(b) Bazgir, A., Ahadi, S., Ghahremanzadeh, R., Khavasi, H.R. and Mirzaei, P. (2010) Ultra Sound Assisted One-Pot Three Component Synthesis of Spiro[Indoline-3,4'pyrazolo[3,4-b]pyridine]-2,6'(1'H)diones in Water. Ultrasonics Sonochemistry, 17, 447-452. http://dx.doi.org/10.1016/j.ultsonch.2009.09.009

[55] (a) Sinha, A.K., Joshi, B.P., Sharma, A., Kumar, V. and Acharya, R. (2007) Microwave Assisted Mild Conversion Natural Dihydrotagetone into 5-Isobutyl,3-methyl-4,5-dihydro-2(3H)-furanone an Analogue of Whisky Lactone. Australian Journal of Chemistry, 60, 124-127. http://dx.doi.org/10.1071/CH06380

(b) Sinha, A.K., Sharma, A. and Joshi, B.P. (2007) One-Pot Two-Step Synthesis of 4-Vinyl Phenols from 4-Hydroxy Benzaldehydes under Microwave Irradiation: A New Perspective on the Classical Knoevenagel-Doebner Reaction. Tetrahedron, 63, 960-965. http://dx.doi.org/10.1016/j.tet.2006.11.023

(c) Kumar, V., Sharma, A., Sharma, M., Sharma, U.K. and Sinha, A.K. (2007) DDQ Catalysed Benylic Acetoxylation of Arylalkanes: A Case of Exquisitely Controlled Oxidation under Sonochemical Activation. Tetrahedron, 63, 97189723. http://dx.doi.org/10.1016/j.tet.2007.07.018

[56] Nagargoje, D., Mandhane, P., Shingote, S., Badadhe, P. and Gill, C. (2012) Ultra Sound Assisted One-Pot Synthesis of Imidazole Derivatives Using Diethyl Bromophosphate as an Oxidant. Ultrasonics Sonochemistry, 19, 94-96. http://dx.doi.org/10.1016/j.ultsonch.2011.05.009

[57] Nikpassnd, M., Mamaghani, M., Shirini, F. and Tabarabaeian, K. (2010) A Convenient Ultrasound Promoted Regioselective Synthesis of Fused Polycyclic 4-Aryl-3-methyl,4,7-dihydro-1H-pyrazolo[3,4b]pyridines. Ultrasonics Sonochemistry, 17, 301-305. http://dx.doi.org/10.1016/j.ultsonch.2009.08.001

[58] Mamaghani, M., Loghmanifar, A. and Taati, M.R. (2011) An Efficient One-Pot Synthesis of New 2-Amino,1,3-thiazolidin-4-ones under Ultrasonic Conditions. Ultrasonics Sonochemistry, 18, 45-48. http://dx.doi.org/10.1016/j.ultsonch.2010.05.009

[59] Zang, H., Su, Q., Mo, Y. and Cheng, B. (2011) Ionic Liquid under Ultrasonic Irradiation towards a Facile Synthesis of Pyrazolone Derivatives. Ultrasonics Sonochemistry, 18, 68-72. http://dx.doi.org/10.1016/j.ultsonch.2010.08.001

[60] (a) Gaplovsky, A., Gaplovsky, M., Toma, S. and Luche, J.L. (2000) Ultrasound Effects on the Photopinacolization of Benzophenone. The Journal of Organic Chemistry, 65, 8444-8447. http://dx.doi.org/10.1021/jo000611+ (b) Rajagopal, R., Jarikote, D.V. and Srinivasan, K.V. (2002) Ultrasound Promoted Suzuki Cross-Coupling Reactions in Ionic Liquid at Ambient Conditions. Chemical Communications, 616-617. http://dx.doi.org/10.1039/b111271f (c) Song, B.A., Zhang, G.P., Yang, S., Hu, D.Y. and Jin, L.H. (2001) Synthesis of N-(4-bromo-2-trifluoromethylphenyl)1-(2-fluorophenyl)-O,O-dialkyl- $\alpha$-aminophosphonates under Ultrasonic Irradiation. Ultrasonics Sonochemistry, 13, 139. http://dx.doi.org/10.1016/j.ultsonch.2005.03.003 